\title{
Correlations of Morphometric Placental Microvessels Parameters with Birth Weight
}

\author{
Dibyajyoti Boruah $^{1 *}$, K Mohanlal ${ }^{2}$, Ajay Malik ${ }^{3}$, Arijit Sen ${ }^{1}$ and Prabal Deb ${ }^{4}$ \\ ${ }^{1}$ Department of Pathology, Armed Forces Medical College, Pune, INDIA \\ ${ }^{2}$ Department of Anatomy, Armed Forces Medical College, Pune, INDIA \\ ${ }^{3}$ Department of Pathology, Army Hospital (R\&R), New Delhi, INDIA \\ ${ }^{4}$ Department of Pathology, Command Hospital (EC),Alipore Rd, Kolkata, INDIA
}

\begin{abstract}
Introduction: Birth weight (BWT) is greatly affected by placental insufficiency and the microvessels configurations. Aims of this study were to evaluate various parameters: villous density (VD), microvessel density (MVD), microvessels per villus (MVPV), mean microvessel caliber (VC), total microvessels boundary density (TVBD), maximum possible total length of microvessels (MP-TVL) and maximum possible total surface area of microvessels (MP-TVSA) in terminal villi of placenta. All the parameters including placental weight (PWT) were correlated with BWT; and MVD and VC of placenta were compared with the human tissues from various lesions.

Materials and methods: Sixty human placentas of uncomplicated term pregnancies ( $\geq 37$ weeks) managed at this tertiary care institute were included. Formalin fixed paraffin embedded sections of placental tissues were used for computer assisted digital image morphometry. H\&E stained sections were used to determine VD and CD34 immuno-stained sections were used for evaluation of vascular parameters in terminal villi. Results: The mean VD was $204 \mathrm{~mm}-2$, the mean MVD was $1314 \mathrm{~mm}-2$, the mean MVPV was 6.6 , the mean MVC was $10.15 \mu \mathrm{m}$, the mean TVBD was $40.64 \mathrm{~mm}-1$, the mean MP-TVL was $626 \mathrm{~km}$ and the mean MP-TVSA was $19.23 \mathrm{~m} 2$. The BWT showed significant $(\mathrm{p}<0.05)$ positive correlations with PWT $(\mathrm{r}=0.690)$, VD $(\mathrm{r}=0.328)$, MVD $(\mathrm{r}=0.408)$, TVBD $(\mathrm{r}=0.280)$, MP-TVL $(\mathrm{r}=0.723)$ and MP-TVSA $(\mathrm{r}=0.723)$; whereas did not show significant correlations with MVPV $(\mathrm{r}=0.185, \mathrm{p}=0.157)$ and $\mathrm{VC}(\mathrm{r}=-0.249$, $\mathrm{p}=0.054)$. MVD in placentas showed strong negative correlation with VC $(r=-0.712)$. MVD in placentas were several times $(\sim 6.3$ times $)$ higher than human tissues; though mean caliber of microvessels in placenta was comparable.
\end{abstract}

Conclusion: PWT, VD, MVD and TVBD in placenta showed significant positive correlations with BWT. Estimated MP-TVL and MP-TSA showed the best correlations with BWT. Placental MVD showed strong negative correlation with VC. Total length of microvessels in the placenta was comparable to that estimated for the fetus.

Keywords: Microvessels in Placenta, Birth Weight, Placental Weight, Morphometry

\section{Introduction}

The placenta develops during pregnancy, attached to the wall of the mother's uterus and connected with the fetus through umbilical cord; provides oxygen and nutrients to the growing fetus and removes waste products from the fetal blood [1]. The growth of the fetus is effected by the placental adequacy and concentration of nutrients / oxygen in the maternal blood supplied to placental region [1]. Terminal villi of the placenta are the main functional components and take important role in fetomaternal transfer of substances. The vast majority of microvessels in placenta are situated in the terminal villi [2]. These microvessels in the placenta provide as a way to exchange of nutrients and metabolic products between mother and fetus [3,4]. The exchange of waste products and nutrients in placenta takes place through the surface of capillaries by diffusion and active transport mechanisms. Hence surface area of microvessel wall is a crucial factor to decide the rate of transportation of substances. Any alteration of capillaries in terminal villi has straight effect on fetomaternal transfusion, fetal growth and development [5]. Development of fetus could be disturbed by placental insufficiency that can result to low birth weight, premature infants, and increased perinatal mortality and morbidity [69]. Placental size and microvessels' quantity increase up to the end of the pregnancy as fetal nutrients requirements increase with gestational duration $[1,10]$.

The concentration, size and function of microvessels in placenta are the area of interest to understand low birth weight, intrauterine growth retardation and early neonatal complications. Total amount of microvessels in a placenta depends on the placental volume and microvessel concentration. The weight of a placenta gives scalar measure of placental volume; positive correlation between birth weight and placental weight/volume was observed by many researchers [11-14]. Stereological and morphometric 
techniques were used by various researchers for quantitative assessment of villi and microvessels in placenta [15-24].

Since fetal growth primarily depends on sufficiency of the placenta; it is interesting to find out the correlations of placental morphometric parameters with birth weight of a baby. In the present study, density of terminal villi (VD), microvessel density (MVD) and mean microvessel caliber (VC) in terminal villi of placenta were evaluated. Microvessels per villus (MVPV), total microvessels boundary density (TVBD), maximum possible total length of microvessels (MP-TVL) and maximum possible total surface area of microvessels (MP-TVSA) were calculated. All these parameters including placental weight (PWT) were correlated with birth weight of the baby (BWT); and found out the parameter displayed the best correlation with BWT. Further, MVD and VC of placenta were compared with the human tissues of different lesions.

\section{Materials and Methods}

The study was conducted on sixty human placentas of uncomplicated term pregnancies managed at this tertiary care institute. Gestational age more than 37 weeks was included in the study. There were 28 cases of low birth weight $(<2.50 \mathrm{~kg})$ with no apparent cause; and 32 cases of normal birth weight $(\geq 2.50 \mathrm{~kg})$. Exclusion criteria for placenta were: intra uterine growth restricted fetuses with known causes, multiple pregnancies, fetuses with congenital anomalies, maternal preeclampsia, maternal diabetes mellitus and infection, placental tumors and trauma, and mother diagnosed with any systemic disorder. Data of BWT, gestational age and mother particulars were available for all the cases included in this study. The study was approved by the Institutional ethical committee.

Weight of the complete placenta (PWT) was recorded for each case after delivery. The placenta were fixed with $10 \%$ formalin and processed for routine paraffin embedding. From each placenta 5 whole thickness tissue blocks were taken from the centre, periphery and intermediate areas. Five micron thick paraffin sections were made, processed and stained by hematoxylin and eosin (H\&E) for microscopic observations. One or two representative sections were selected for CD34 immuno-staining and morphometry. $H \& E$ stained sections were used to determine number density of terminal villi (VD) and CD34 immuno-stained sections were used for evaluation of vascular parameters in terminal villi.

\section{Morphometry}

Representative blocks of formalin-fixed paraffin-embedded tissue were selected; 5-micrometer thick sections cut and immunohistochemical (IHC) staining performed by LSAB technique using mouse anti-human CD34 monoclonal antibody and counter stained by hematoxylin. Vascular morphometric analysis was performed on microscopic image of CD34 immunostained sections. Sections were scanned under the microscope at low power (x100) magnification, searched area of the greatest number of distinctly CD34 immuno-stained microvessels in terminal villi of placenta and selected for imaging. Five selected microscopic fields of each sample at 400x magnification were recorded by a computerized digital photomicrograph system (Dewinter Optical Inc. with Digi Eye 330 digital photomicrography camera and Biowizard 4.1 Image analysis software) for morphometry. Images were processed using the software to get sharp microvessel boundary before performing the measurement. Similarly, representative H\&E stained sections were scrutinized under the microscope at low power magnification, searched region of the largest number of distinct terminal villi and captured five fields at high power(x400) magnification for the determination of VD.

The measuring scale of image analysis software was properly calibrated with the standard scale, as per instruction given in the software manual. Microvessel counting and calibre measurement were performed on the images of recorded five fields (total area $=0.289 \mathrm{~mm}^{2}$ ). Microvessels count was used to calculate MVD. The microvessels which appeared circular and elliptical in the section were used to evaluate microvessel caliber [25]. For the elliptical cases, the minor axis of the vessel was considered as its caliber. The microvessel caliber was measured by tracing the cursor of the mouse using the software [Figure1]. After measurement, the data was transferred to MS-Excel sheet for further analysis.

Counting of terminal villus was done on the five recorded fields of H\&E stained section for each sample. The total villus count was used to calculate VD.

Parameters: VD, MVD, MVPV and TVBD were calculated using the following relations:

$\mathrm{VD}=($ Total number of villi in 5 high power fields $) /($ Total area of the 5 fields)

MVD $=($ Total number microvessels in 5 high power fields $)$ / (Total area of the 5 fields)

$\mathrm{MVPV}=(\mathrm{MVD} / \mathrm{VD})$

$\mathrm{TVBD}=(\pi \times \mathrm{MVD} \times \mathrm{VC})$

Maximum possible total length of microvessels (MP-TVL) for each placenta was estimated from the information of MVD and volume of placenta using stereological analogy. Volume of placenta is given by $[(\mathrm{PWT}) /$ (density of placenta $(\rho))$ ]. Hence, 
MP-TVL $=($ MVD $\times \mathrm{PWT}) / \rho$

Similarly maximum possible total surface area of microvessels (MP-TVSA) for each placenta was estimated from TVBD and volume of placenta using stereological analogy:

MP-TVSA $=($ TVBD $\times$ PWT $) / \rho$

In this study we consider $\rho=10^{3} \mathrm{~kg} / \mathrm{m}^{3}$ for calculation of MP-TVL and MP-TVSA.

\section{Statistical Analysis}

SPSS17.0 (Statistical Package for Social Sciences) software programme was used in statistical analysis. For each sample, BWT and PWT were recorded; MVD and mean VC with SD were determined; TVBD, MP-TVL and MP-TVSA were calculated. The mean values of these parameters with standard deviation (SD) and range were evaluated for the 60 studied samples. The statistical correlations of the analyzed parameters for all samples with BWT; and between each pair of the parameters were investigated; and Pearson correlation coefficients ' $r$ ' were calculated. Mean MVD and VC of placenta were compared with the tissues of different lesions [normal brain $(n=10)$, normal gingival tissue $(n=30)$, normal colon $(n=30)$, dermis in psoriasis $(n=50)$, gliomas $(n=30)$, invasive ductile carcinoma breast $(n=55)$ and colorectal carcinoma $(n=30)$ ] studied by us previously using the same method and image analyzing system.

\section{Results}

Screen shot of a CD34 immuno-stained histological section used for evaluation of VC and MVD are shown in Figure1. Mean values with standard deviation and range of the parameters of 60 studied placentas are presented in Table1. Table 2 shows the Pearson correlation coefficients between pairs of studied parameters. Figure.2(A-H) represent the scatter plots of PWT, VD, MVD, MVPV, VC, TVBD, MP-TVL and MP-TVSA with BWT for all samples. In each figure, linear regression line and Pearson correlation coefficient (r) with significance ( $\mathrm{p}$ value) are presented.

The mean gestational period was 40.0 weeks (range: $37.0-$ 44.0 weeks) and the mean mother age was 23 years (range: 18-30years) at the time of delivery. The mean BWT was $2.56 \mathrm{~kg}$ (range: $1.25-3.36 \mathrm{~kg}$ ) and the mean PWT was $0.47 \mathrm{~kg}$ (range:0.31-0.74 kg). The mean VD was 204 $\mathrm{mm}^{-2}$ (range: $139 \mathrm{~mm}^{-2}-303 \mathrm{~mm}^{-2}$ ) and the mean MVD was $1314 \mathrm{~mm}^{-2}$ (range: $767 \mathrm{~mm}^{-2}-1937 \mathrm{~mm}^{-2}$ ). The mean MVPV was 6.6 (range: 4.3 -9.3). and the mean MVC was $10.15 \mu \mathrm{m}$ (range: $6.86 \mu \mathrm{m}-16.56 \mu \mathrm{m}$ ). The mean TVBD was $40.64 \mathrm{~mm}^{-1}$ (range: $26.04-53.84 \mathrm{~mm}^{-1}$ ), the mean MP-
TVL was $626 \mathrm{~km}$ (range: $290-1113 \mathrm{~km}$ ) and the mean MPTVSA was $19.23 \mathrm{~m}^{2}$ (range: $9.35-35.55 \mathrm{~m}^{2}$ ).

Correlations of BWT with other parameters:

Investigation was done to correlate the studied parameters with BWT (Fig.2A-H). The BWT showed significant $(\mathrm{p}<0.05)$ positive correlations with PWT $(\mathrm{r}=0.690)$ (Fig.2A), VD (r=0.328) (Fig.2B), MVD ( $\mathrm{r}=0.408)$ (Fig2C), TVBD ( $\mathrm{r}=0.280)$ (Fig.2F), MP-TVL ( $\mathrm{r}=0.723)$ (Fig2G) and MP-TVSA $(\mathrm{r}=0.723)(\mathrm{Fig} 2 \mathrm{H})$; whereas did not show significant correlations with MVPV $(r=0.185, \mathrm{p}=0.157)$ (Fig.2D) and VC (r=-0.249, p=0.054) (Fig2E).

\section{Correlations of MVD, VC and VD}

Fig3 showed scatter plot of MVD versus VC with linear regression and MVD exhibited strong negative correlation with $\mathrm{VC}(\mathrm{r}=-0.712)$.

MVD showed significant positive correlations with $\mathrm{VD}(\mathrm{r}=0.586), \operatorname{MVPV}(\mathrm{r}=0.600)$, TVBD $(\mathrm{r}=0.501)$, MPTVL $(r=0.720)$ and MP-TVSA $(r=0.385)$; but did not show significant correlation with PWT $(\mathrm{p}>0.05)$.

VC showed significant negative correlations with $\mathrm{VD}(\mathrm{r}=-$ 0.329), MVPV( $\mathrm{r}=-0.528)$, MP-TVL $(\mathrm{r}=-0.578)$; but did not showed significant correlations with PWT, TBVD and MPTVSA ( $>>0.05)$.

VD showed significant positive correlations TVBD $(\mathrm{r}=0.374)$, MP-TVL $(\mathrm{r}=0.445)$ and MP-TVSA(r=0.297) and negative correlation with $\operatorname{MVPV}(\mathrm{r}=-0.282)$. But VD did not show any correlation with PWT.

\section{Comparison MVD and VC of Placenta with other Human Tissues}

Fig4(A) and Fig4(B) show the mean with SD of MVD and $\mathrm{VC}$ of normal brain $(\mathrm{n}=10)$, normal gingival tissue $(n=30)$, normal colon $(n=30)$, dermis in psoriasis $(n=50)$, gliomas $(n=30)$, invasive ductile carcinoma breast $(n=55)$ and colorectal carcinoma $(n=30)$ with the presently studied placenta( $n=60)$. The mean values of MVD and VC with $\mathrm{SDs}$, ratios of mean MVD and mean $\mathrm{VC}$ of placentas to various human tissues are presented in Table3. The mean MVD of placentas was 7.18 times higher than normal brain tissues[26], 3.86 times higher than normal gingival tissues[27], 8.16 times higher than normal colons, 7.10 times higher than dermis with psoriasis[25], 5.21 times higher than gliomas of various grades[26], 6.57 times higher than invasive ductile carcinomas breast and 5.76 times higher than colorectal carcinomas. The mean VC of placentas was 1.48 times higher than normal brain tissues[26], 1.62 times higher than normal gingival 
tissues[27], 1.49 times higher than normal colons, 0.86 times lower than dermis with psoriasis[29], 0.88 times lower than gliomas of various grades[26], 0.85 times lower than invasive ductile carcinomas breast and 0.75 times lower than colorectal carcinomas.

\section{Relation between Total Length of Microvessels in} Placenta and Baby

In this study it was observed that the ratio of BWT to PWT was comparable to the ratio of MVD in placenta to normal human tissue. Hence it is very likely that a meaningful relation may exist between the total length of microvessels in placenta and baby. Hypothetically, we proposed that the MP-TVL in placenta was equal to MP-TVL in baby; i.e.

$\left(\mathrm{MVD}_{\mathrm{PLAC}} \times \mathrm{PWT}\right) / \rho_{\mathrm{PLAC}}=\left(\mathrm{MVD}_{\mathrm{BABY}} \times \mathrm{BWT}\right) / \rho_{\mathrm{BABY}}$

Where, $\mathrm{MVD}_{\mathrm{PLAC}}$ and $\mathrm{MVD}_{\mathrm{BABY}}$ are average MVD of the placenta and the baby at birth respectively. $\rho_{\mathrm{PLAC}}$ and $\rho_{\mathrm{BABY}}$ are the densities of placenta and baby respectively.

Considering $\rho_{\mathrm{PLAC}}=\rho_{\mathrm{BABY}}$, we obtained $\mathrm{MVD}_{\mathrm{BABY}}=$ $\left[\left(\mathrm{MVD}_{\mathrm{PLAC}} \times \mathrm{PWT}\right) /(\mathrm{BWT})\right]$. Using this relation $\mathrm{MVD}_{\mathrm{BABY}}$ was estimated for each case. The mean $\mathrm{MVD}_{\mathrm{BABY}}$ was $244 \mathrm{~mm}^{-2}$ (SD: $55 \mathrm{~mm}^{-2}$, range: $146-360 \mathrm{~mm}^{-2}$ ), and it was comparable to the MVD of other normal human tissues incorporated in this study (Table3). Using this mean $\mathrm{MVD}_{\mathrm{BABY}}\left(=244 \mathrm{~mm}^{-2}\right)$, the MP-TVL in baby was calculated for each case, and presented with MP-TVL in placenta in Figure5. Using paired Students t-test we observed that there is no significant difference between MP-TVL in baby and placenta $(\mathrm{p}=0.9021)$. Hence, validity of the proposed hypothesis, "Total length of microvessels in a baby is comparable to the total length of microvessels in the placenta" is very likely.

Table 1: Mean value with standard deviation and range of birth weight (BWT), placental weight(PWT), villous density(VD) and placental microvessels parameters of 60 studied cases.

\begin{tabular}{|c|c|c|}
\hline SI No & Parameters (unit) & Mean \pm SD (min-max) \\
\hline 1 & BWT $(\mathrm{kg})$ & $2.56 \pm 0.51(1.25-3.36)$ \\
\hline 2 & PWT $(\mathrm{kg})$ & $0.47 \pm 0.11(0.31-0.74)$ \\
\hline 3 & $\operatorname{VD}\left(\mathrm{mm}^{-2}\right)$ & $204 \pm 36(139-303)$ \\
\hline 4 & MVD $\left(\mathrm{mm}^{-2}\right)$ & $1314 \pm 276(767-1937)$ \\
\hline 5 & MVPV & $6.6 \pm 1.2(4.3-9.3)$ \\
\hline 6 & VC $(m i c r o n)$ & $10.15 \pm 2.03(6.86-16.56)$ \\
\hline 7 & TVBD $\left(\mathrm{mm}^{-1}\right)$ & $40.64 \pm 5.82(26.04-53.84)$ \\
\hline 8 & MP-TVL $\left(\mathrm{km}^{-1}\right)$ & $626 \pm 199(290-1113)$ \\
\hline 9 & MP-TVSA $\left(\mathrm{m}^{2}\right)$ & $19.23 \pm 5.01(9.35-35.55)$ \\
\hline
\end{tabular}

Table 2: Pearson correlation coefficients between pairs of studied parameters of all 60 cases.

\begin{tabular}{|c|c|c|c|c|c|c|c|c|c|}
\hline & BWT & PWT & VD & MVD & MVPV & VC & TVBD & MP-TVL & MP-TVSA \\
\hline BWT & 1.000 & & & & & & & & \\
\hline PWT & $0.690^{* *}$ & 1.000 & & & & & & & \\
\hline VD & $0.329^{*}$ & 0.100 & 1.000 & & & & & & \\
\hline MVD & $0.408^{* *}$ & 0.142 & $0.586^{* *}$ & 1.000 & & & & & \\
\hline MVPV & 0.185 & 0.102 & $-0.282^{*}$ & $0.600^{* *}$ & 1.000 & & & & \\
\hline VC & -0.249 & -0.208 & $-0.329^{*}$ & $-0.712^{\star *}$ & $-0.528^{\star *}$ & 1.000 & & & \\
\hline TVBD & $0.280^{*}$ & -0.015 & $0.374^{* *}$ & $0.501^{* *}$ & 0.220 & 0.216 & 1.000 & & \\
\hline MP-TVL & $0.723^{* *}$ & $0.778^{* *}$ & $0.445^{\star *}$ & $0.720^{\star *}$ & $.429^{* *}$ & $-0.578^{* *}$ & $0.305^{*}$ & 1.000 & \\
\hline MP-TVSA & $0.723^{* *}$ & $0.833^{* *}$ & $0.297^{*}$ & $0.385^{\star *}$ & 0.189 & -0.057 & $0.527^{* *}$ & $0.823^{* *}$ & 1.000 \\
\hline
\end{tabular}


Table 3: Comparison of microvessel densities and microvessel calibers of placenta with three types of normal tissues (brain, gingival tissue and colon) and four different types of diseased lesions (dermis in psoriasis, gliomas, invasive ductile carcinoma breast and colorectal carcinoma). All the tissues considered for comparison were previously studied by us using same method for evaluation of MVD and VC. Mean MVD of placenta was 3.86 to 8.16 (average $=6.40$ ) times of other tissues. Mean VC of placenta was larger than normal tissues (1.30 to 1.48 times) and smaller than diseased tissues (0.75 to 0.88 ).

\begin{tabular}{|c|c|c|c|c|c|}
\hline $\begin{array}{l}\text { SI } \\
\text { No }\end{array}$ & Tissue (no of sample) & $\begin{array}{c}\text { MVD } \pm \text { SD } \\
\left(\mathrm{mm}^{-1}\right)\end{array}$ & $\begin{array}{l}\text { VCE SD } \\
\text { (micron) }\end{array}$ & $\begin{array}{l}\text { MVD }_{\text {PLAC }} l \\
\text { MVD }_{\text {TISSUE }}\end{array}$ & $\begin{array}{l}V_{C_{\text {PLAC }}}{ }^{\prime} \\
V_{\text {TISSUE }}\end{array}$ \\
\hline 1 & Placenta (60) & $1314 \pm 276$ & $10.15 \pm 2.03$ & 1.00 & 1.00 \\
\hline 2 & Normal brain(10) & $183 \pm 38$ & $6.87 \pm 0.75$ & 7.18 & 1.48 \\
\hline 3 & Normal gingival tissue (30) & $340 \pm 33$ & $6.25 \pm 0.96$ & 3.86 & 1.62 \\
\hline 4 & Normal colon $(30)$ & $161 \pm 33$ & $6.80 \pm 1.34$ & 8.16 & 1.49 \\
\hline 5 & Dermis in psoriasis(50) & $185 \pm 57$ & $11.82 \pm 2.45$ & 7.10 & 0.86 \\
\hline 6 & Gliomas(30) & $252 \pm 92$ & $11.53 \pm 2.68$ & 5.21 & 0.88 \\
\hline 7 & Invasive ductile carcinoma breast (55) & $200 \pm 36$ & $11.91 \pm 1.37$ & 6.57 & 0.85 \\
\hline 8 & Colorectal carcinoma $(30)$ & $228 \pm 68$ & $13.54 \pm 2.45$ & 5.76 & 0.75 \\
\hline
\end{tabular}

$\mathrm{MVD}=$ microvessel density, $\mathrm{VC}=$ microvessel caliber, $\left(\mathrm{MVD}_{\mathrm{PLAC}} / \mathrm{MVD}_{\text {TISSUE }}\right)=$ ratio of mean MVD of placenta to tissue, $\left(\mathrm{VC}_{\mathrm{PLAC}} / \mathrm{VC}_{\mathrm{TISSUE}}\right)=$ ratio of mean $\mathrm{VC}$ of placenta to tissue

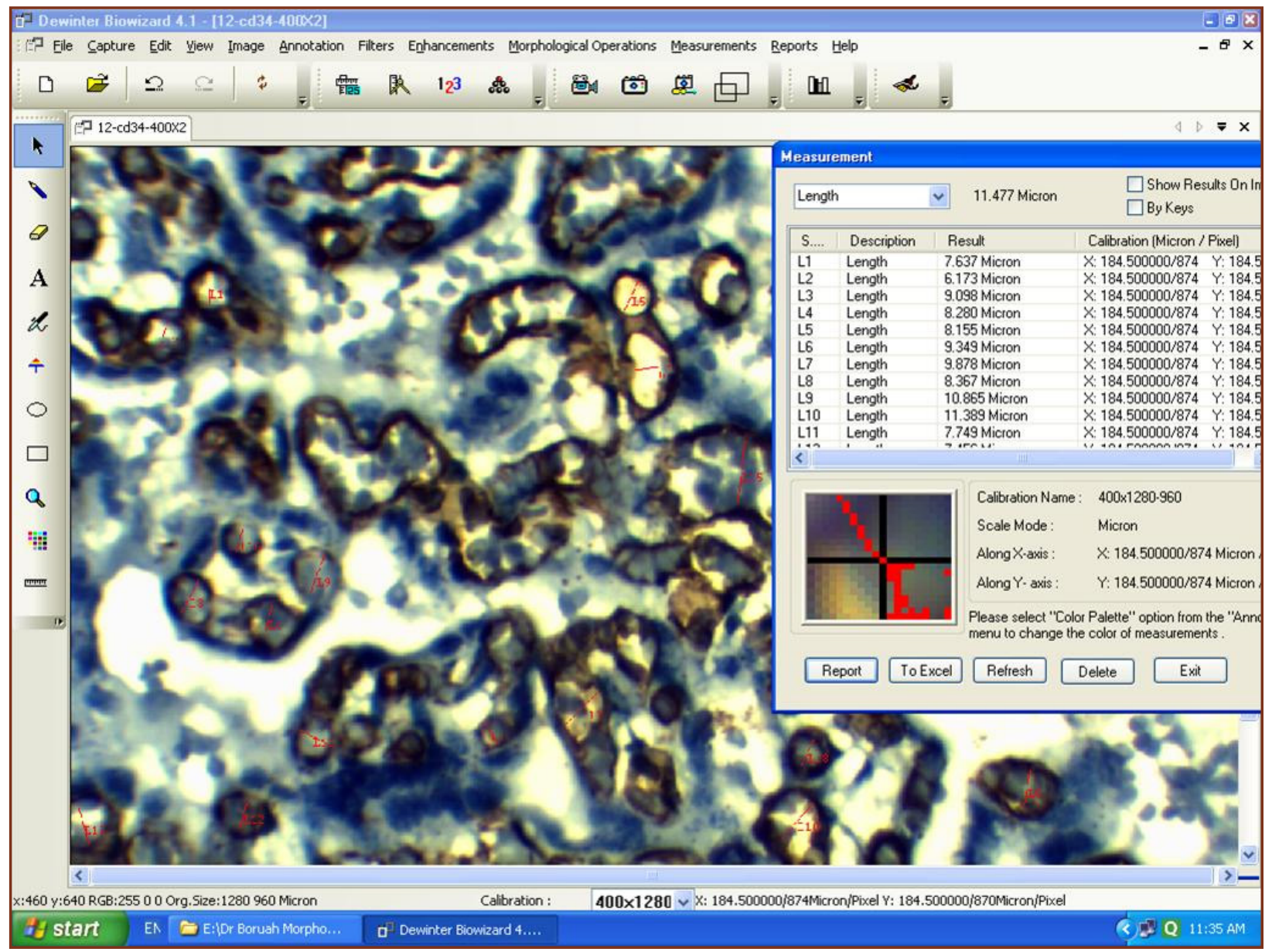

Fig. 1: CD34 immuno-stained histological section (400 X original magnifications) in terminal villi of a placenta used for evaluation of VC and counting of microvessels by image analyzing software. VC of the microvessels appeared circular and elliptical was measured by tracing the mouse of the computer using the software. 


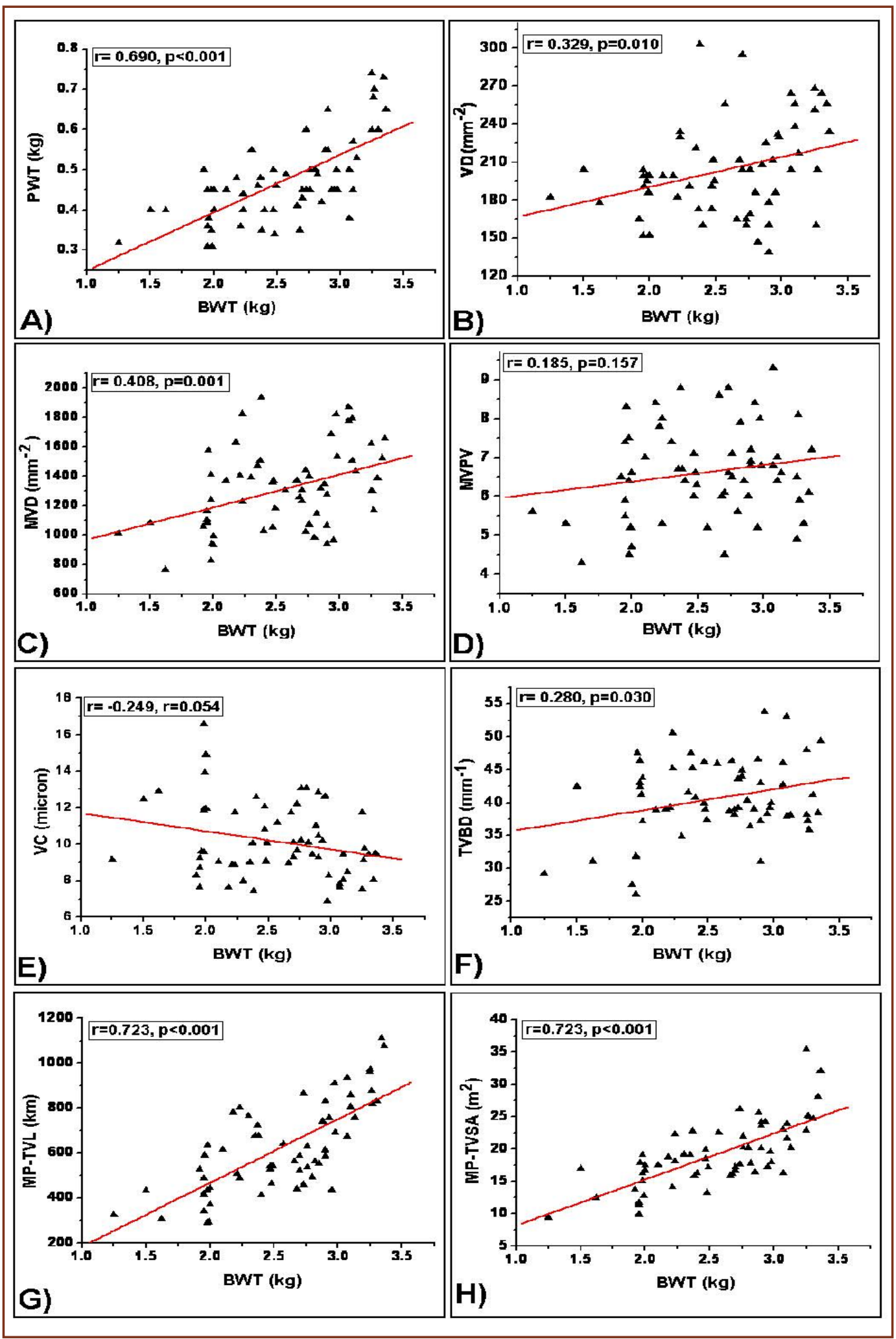

Fig. 2: (A-H). Scatter plots of A) PWT, B) VD in placenta, C) placental MVD, D) MVPV in placenta, E) placental VC, F) TVBD in placenta, G) MP-TVL in placenta and H) MP-TVSA in placenta with BWT for all 60 cases. Solid line in each figure represents the regression line. Pearson correlation coefficient ( $r$ ) with significance ( $p$ value) is presented in each figure. 


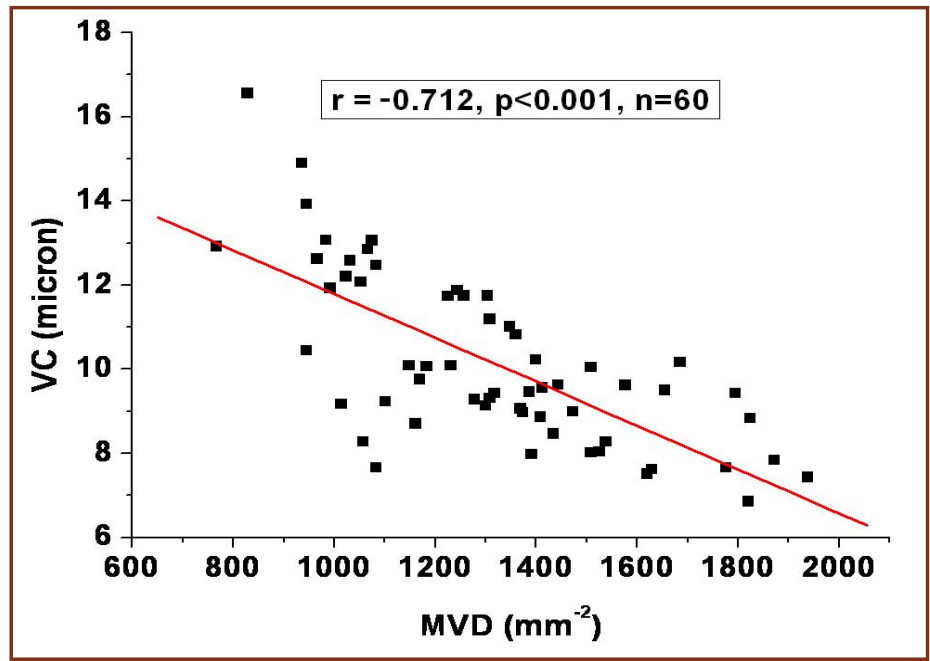

Fig. 3: Scatter plot of MVD versus VC with linear regression. Pearson correlation coefficient ( $r$ ) with significance ( $p$ value) is presented in the box.

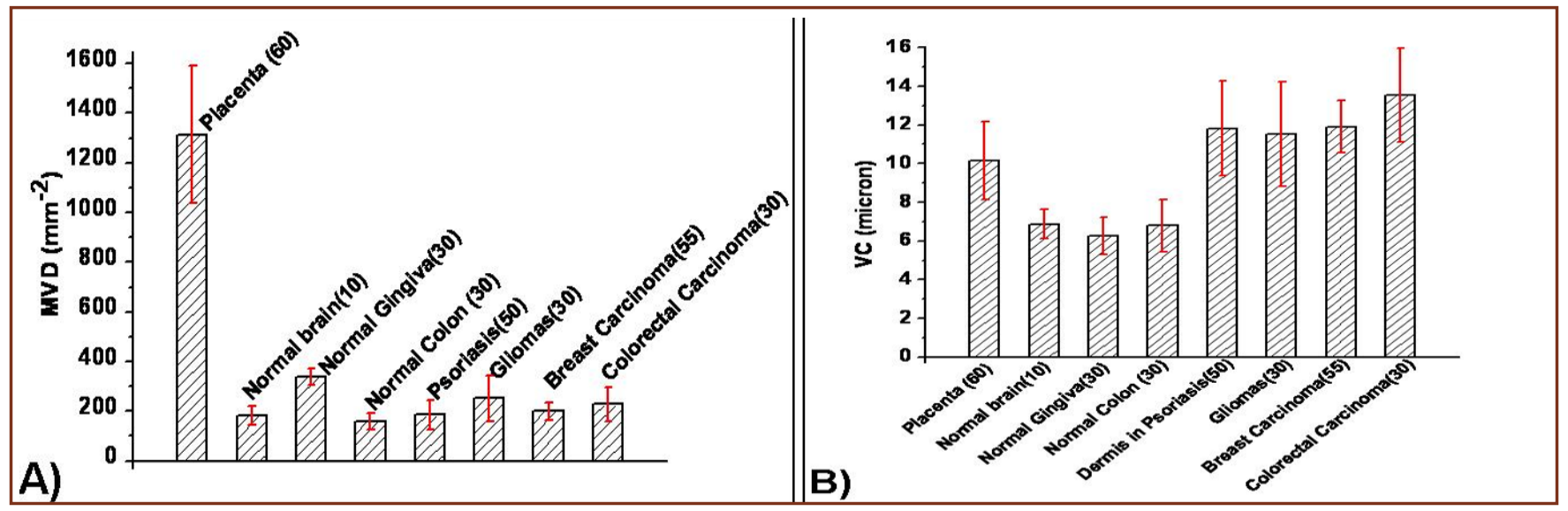

Fig. 4: (A-B). The mean value with SD (denoted by the error bar) of A) MVD and B) VC in normal brains( $\mathrm{n}=10$ ), normal gingival tissues $(n=30)$, normal colons $(n=30)$, dermis in psoriasis $(n=50)$, gliomas $(n=30)$, invasive ductile carcinomas breast $(n=55)$ and colorectal carcinomas $(n=30)$ with the presently studied placentas $(n=60) . n=$ number of samples.

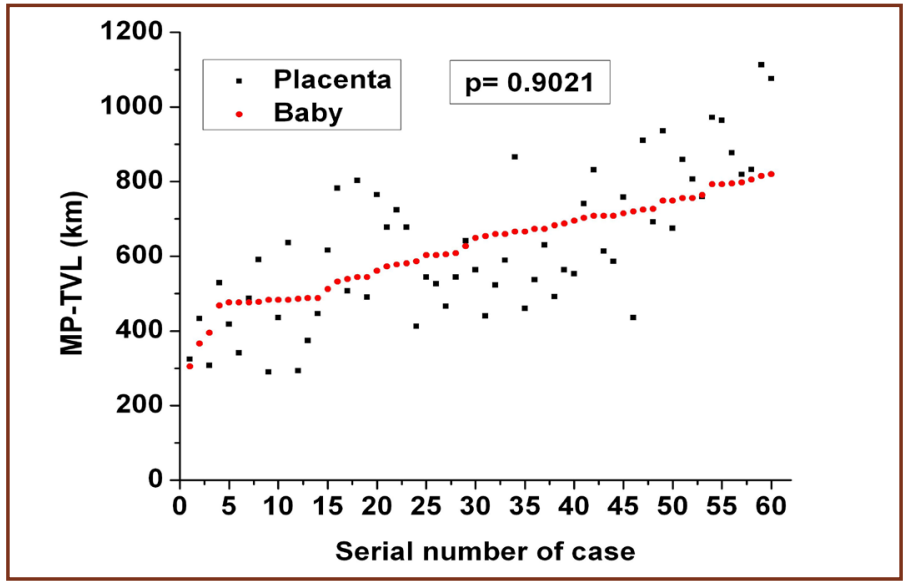

Fig. 5: Scatter plots of estimated MP-TVL in babies considering MVDBABY $=244 \mathrm{~mm}-2$ and MP-TVL in placentas for the 60 studied cases. $p$ value of paired t-test is shown in box. 


\section{Discussion}

Birth weight in termed pregnancy is influenced by various factors like: placental insufficiency and concentration of substances in the maternal blood supplied to placental region during gestational period, maternal health status/ disease, ethnic and geographical variations, and genetic expression [3-5]. Low birth weight due to pre-eclampsia and fetal growth restriction are connected with fetal hypoxia resulting partially from alterations in the growth and development of the placental villi and their underlying vasculature $[15,28]$. Whereas, reduced uteroplacental blood supply leads to low birth weight in pre-eclampsia in many cases [29]. Further, altered intervillous space partial pressure of oxygen in impaired uteroplacental blood flow in intrauterine growth restricted pregnancies, is associated with reduced transporter activity [30]. In a morphometric placental study, Egbor et al suggested two separate sets of pre-eclampsia: early-onset $(<34$ weeks) and late-onset $(>34$ weeks); abnormal placental morphology was associated with the isolated early-onset pre-eclampsia; whereas late-onset pre-eclampsia was a maternal disorder and not a placental disease[15]. In many cases, the birth weights vary in termed pregnancies without any known cause. Study of placental vasculature could provide useful input to understand such conditions [8].

In the present study, the role of the placental microvessels parameters in the final growth of fetus (i.e. birth weight) using morphometry was assessed; where only termed pregnancies without any known maternal disease/disorder were included. Placentas from underweight infants (without any known abnormalities) to normal birth weight were incorporated in this study. Microvessels parameters were evaluated in terminal villi of placenta, and the results were extrapolated to estimate information for whole placenta. We did not find significant correlation of BWT with MVPV of placenta, but found mild positive correlation with VD of placenta. Possibility of increase in VD with BWT, suggested significant positive correlation of MVD in placenta with BWT; and we observed the same. TVBD was calculated from MVD and VC and it signified the available microvessels surface area in unit volume of placenta. In this study, VC did not show significant correlation with BWT, but TVBD of placenta showed significant positive correlation with BWT like MVD. This revealed that, the parameters related to the concentration of microvessels and terminal villi in placenta have definite influence on the growth of the fetus and hence birth weight.

Placenta weight is a reliable predictor of postnatal health and it shows positive correlations with birth weight [1114]. Haeussner et al showed that shape and size of the placenta have different functional meanings and can be determined independently; where birth weight correlates with size (weight) but not shape of the normal placenta [11]. In our study, we also observed highly significant positive correlation of BWT with PWT. To explain this fact, we defined and calculated two parameters: MP-TVL and MPTVSA. MP-TVL and MP-TVSA in a placenta increase with increase of PWT and MVD/TVBD. As expected, strong positive correlations MP-TVL and MP-TVSA were observed with BWT. Since, the value of PWT and MVD/TVBD were included for the estimation of MP-TVL and MP-TVSA; hence we observed the best correlations of them with BWT. In fetomaternal transportation, total surface area of microvessels in placenta takes an important role. Exchange of nutrients and waste products in placenta takes place through the capillary surface. The rate of transportation of substances is directly proportional to the total surface area of the placental microvessels, besides other factors like: concentration gradients of substances between maternal and fetal blood, and permeability of capillary surface. For uninterrupted growth of a fetus, the placenta should provide optimal quantity of nutrients and effectively discharge waste products [4]. When the placenta is not able to supply required substances to the fetus, its growth will be restricted [8]. In this study, BWT showed the best correlations with MP-TVL and MPTVSA, hence these two parameters can be considered to evaluate sufficiency of a placenta.

MVD and VC in placenta showed strong negative correlation with each other. Hence with the decrease of MVD, VC in placenta increases and vice versa. When MVD in placenta decreases, the available microvessels surface area will be reduced and may hamper fetomaternal transportation; to counter balance this adverse effect the VC likely to increases such cases to maintain optimal transfer of nutrients to the fetus.

The microvessel density in a placenta was found several times higher than normal human tissue; though mean caliber of microvessels in placenta was comparable. Determination of microvessels parameters using histomorphometry in infants is difficult due to unavailability of such tissue. Hence, we estimated MVD in infant from the MVD of placenta, assuming the maximum possible total length of microvessels in baby and placenta was equal. We found that the estimated MVD in infant was comparable to the MVD of different human tissue found by same morphometric system. Further we calculated MP-TVL in infants using the estimated MVD and found that it was not significantly different from the MP-TVL of placenta. Hence, from our study it could be proposed that the total microvessels length in placenta is nearly equal that in the 
baby. For efficient growth of a fetus, the exchange rate of substance through the microvessels' surface in placental region should be equal to the exchange rate in the tissue of the fetus. To fulfill this condition, total length of microvessels in placenta and fetus should be comparable; and we find similar indication in our study.

\section{Conclusion}

Growth of a fetus is greatly affected by the microvessels constitution in placenta. Morphometric placental microvessels parameters are very useful to assess and understand the effectiveness of placenta. PWT, VD, MVD and TVBD in placenta showed significant positive correlations with BWT. MVPV and VC in placenta did not show significant positive correlation with BWT. Estimated MP-TVL and MP-TSA showed strong positive correlations with BWT and they were the best parameters for the assessment of placental sufficiency. Placental MVD showed strong negative correlation with VC. MVD in a placenta was several times higher than normal human tissue; though mean caliber of microvessels in placenta was comparable. Total length of microvessels in the placenta was comparable to that estimated for the fetus.

\section{Acknowledgements}

Authors acknowledge the technicians of the Department of Pathology of their institute for their support in preparing the sections.

\section{References}

1. Kaufmann P, Mayhew TM, Charnock-Jones DS. Aspects of human fetoplacental vasculogenesis and angiogenesis. II. Changes during normal pregnancy. Placenta 2004; 25: 114-126.

2. Žigic Z, Markovic S, Grbeša D, Ramic S, Halilovic A. Quantitative research of capillaries in terminal villi of mature placentae. Bosnian journal of basic medical sciences 2010; 10 (2): 147-152.

3. Reynolds LP and Redmer DA. Angiogenesis in the Placenta. Biology of Reproduction 2001; 64:1033-1040.

4. Murray AJ. Oxygen delivery and fetal-placental growth: Beyond a question of supply and demand? Placenta 2012; 33: e16-e22.

5. Kingdom J, Huppertz B, Seaward G, Kaufmann P. Development of the placental villous tree and its consequence fetal growth. Eur. J. Obstet. Gynecol. Reprod. Biol. 2000; 92(I): 35-43.

6. Maulik D, Evans JF, Ragolia L. Fetal growth restriction: Pathogenic mechanisms. Clin Obstet Gynecol 2006; 49: 219-227.

7. Arroyo JA, Winn VD. Vasculogenesis and angiogenesis in the IUGR placenta. Semin Perinatol 2008; 32: 172-177.
8. Ahmed A, Perkins J. Angiogenesis and intrauterine growth restriction. Baillieres Best Pract Res Clin Obstet Gynaecol 2000; 14: 981-998.

9. Barut F, Barut A, Gun BD, Kandemir NO, Harma MI, Harma $\mathrm{M}$ et al. Intrauterine growth restriction and placental angiogenesis. Diagnostic Pathology 2010; 5:24. http://www. diagnosticpathology.org/content/5/1/24.

10. Lee MY, Huang JP, Chen YY, Aplin JD, Wu YH, Chen CY et al. Angiogenesis in differentiated placental multipotent mesenchymal stromal cells is dependent on integrin alpha5beta1. PLoS One 2009; 4: e6913.

11. Haeussner E, Schmitz C, Von-Koch F, Frank HG. Birth weight correlates with size but not shape of the normal human placenta. Placenta 2013; 34: 574-582.

12. Biswas S, Chattopadhaya JC, Ghosh SK. Volume of placenta and chorionic villi as indicator of intrauterine growth restriction of fetuses. J. Anat Soc. India 2007; 56(2): 25-29.

13. Anas IY and Saleh MS. Birth weight and placental weight correlation of Fulani ethnic group Parturients in Nigeria. British Journal of Medicine \& Medical Research 2014; 4(4): 1049-1055.

14. Virupaxi RD, Potturi BR, Shirol VS, Desai SP, Hukkeri VB. Morphology of placenta and its relation with small for date babies in 950 live births. Recent Research in Science and Technology 2011; 3(2): 123-126.

15. Egbor M, Ansari T, Morris N, Green C, Sibbons P. Morphometric placental villous and vascular abnormalities in early- and late-onset pre-eclampsia with and without fetal growth restriction. BJOG 2006; 113: 580-589.

16. Jirkovská M, Kubínová L, Janáček J, Kaláb J. 3-D study of vessels in peripheral placental villi. Image Anal Stereol 2007; 26: 165-168.

17. Mayhew TM and Burton GJ. Stereology and its impact on our understanding of human placental functional morphology. Microscopy Research and Technique1997; 38:195-205.

18. Mayhew TM. Stereological studies on fetal vascular development in human placental villi Image. Anal Stereol 2003; 22: 49-56.

19. Mayhew TM. Stereology and the Placenta: Where's the Point? - A Review. Placenta 2006; 27: Supplement A, Trophoblast Research(Vol. 20): S17-S25.

20. Coelho TM, Sass N, Camano L, Moron AF, Mattar R, Stávale JN et al. Microvessel density in the placental bed among preeclampsia patients. Sao Paulo Med J. 2006; 124(2): 96-100.

21. Biswas S, Ghosh SK and Chhabra S. Surface area of chorionic villi of placentas: An index of intrauterine growth restriction of fetuses. J. Obstet. Gynaecol. Res.2008; 34: 487-493.

22. Calderon IMP, Damasceno DC, Amorin RL, Costa RAA, Brasil MAM, Rudge MVC. Morphometric study of placental villi and vessels in women with mild hyperglycemia or 
gestational or overt diabetes. Diabetes Research and Clinical Practice 2007; 78: 65-71.

23. Mayhew TM and Jairam IC. Stereological comparison of 3D spatial relationships involving villi and intervillous pores in human placentas from control and diabetic pregnancies. Anat. 2000; 197: 263-274.

24. Mayhew TM. A stereological perspective on placental morphology in normal and complicated pregnancies. J. Anat. 2009; 215: 77-90.

25. Boruah D, Moorchung N, Vasudevan B, Malik A, Chattarjee M. Morphometric study of microvessels, epidermal characteristics and inflammation in psoriasis vulgaris with their correlations. Indian Journal of Dermatology, Venereology and Leprosy 2013: 79(2), 216-223.

26. Boruah D, Deb P, Srinivas V, Mani NS. Morphometric study of nuclei and microvessels in gliomas and its correlation with grades. Microvascular Research2014; 93: 52-61.
27. Prakash P, Rath S, Mukherjee M, Malik A, Boruah D, Sahoo NK et al. Comparative evaluation of the marginal gingival epithelium in smokers and non smokers- A Histomorphometric \& Immunohistochemical study. International Journal of Periodontics \& Restorative Dentistry2014. 34(6), 781-786.

28. Mayhew TM, Ohadike C, Baker PN, Crocker IP, Mitchell C, Ong SS. Stereological investigation of placental morphology in pregnancies complicated by pre-eclampsia with and without intrauterine growth restriction. Placenta 2003; 24: 219-26.

29. Long PA, Abell DA, Beischer NA. Fetal growth retardation and pre-eclampsia. Br J Obstet Gynaecol 1980; 87: 13-18.

30. Nelson DM, Smith SD, Furescz TC, Sadovsky Y, Ganapathy $\mathrm{V}$, Parvin CA et al. Hypoxia reduces the expression and function of system A amino acid transporters in cultured term human trophoblasts. Am J Physiol Cell Physiol 2003;284(2):C310-C315.

*Corresponding author:

Dr Dibyajyoti Boruah, Scientist 'D', Department of Pathology, Armed Forces Medical College, Pune - 411040, INDIA

Phone: +91 9823553654

Email: dibyajyotibh@yahoo.co.uk

Financial or other Competing Interests: None.

Date of Submission : 18.11.2017

Date of Acceptance : 24.11.2017

Date of Publication : 10.12.2017

Annals of Advanced Medical Science, Vol. 1; Issue 1, October-December 2017 